\title{
Health is the Motive and Digital is the Instrument
}

\section{S. Seethalakshmi* and Rahul Nandan}

\begin{abstract}
The coronavirus crisis has seen an unprecedented response from India and the world. If the viral outbreak has exposed gross inadequacies in the healthcare systems of nations both rich and poor, it has stirred a digital healthcare revolution that has been building since the past decade. We have seen how this new era of digital health evolved over the years since healthcare started getting increasingly unaffordable in the western countries forcing a relook in their strategies to explosion of digital innovations in mobile telephony and applications, internet, wearable devices, artificial intelligence, robotics, big data and genomics. The single biggest trigger for the digital shift has indeed been the COVID-19 pandemic this year, more so in India with astonishing response from the private enterprise and the proactive push from the government so evident. However, the full potential of this digital revolution cannot be realized as long as core structural reforms in public healthcare do not take place along with significant boost in digital infrastructure. The way digital technologies have helped facilitate strategy and response to the global pandemic and with predictions of more zoonotic outbreaks impending in the coming years, it has become imperative for the world to increasingly adopt and integrate digital innovations to make healthcare more accessible, interconnected and affordable.
\end{abstract}

Healthcare is realigning in response to COVID19 like never before. The health ecosystem across the world and particularly in India is witnessing a paradigm digital shift to ensure accessibility, affordability and quality care at a time when the viral outbreak has exposed the chronic state of our country's under-funded and ill prepared healthcare infrastructure.

Though COVID-19 pandemic has heralded a new era of digital healthcare across nations, rich and poor, the digital health storm has been brewing over the past decade owing to a host of factors, both welcoming and worrying.

First, the extremely high cost of healthcare in many developed economies, particularly in the US, coupled with low health outcomes have led them to increasingly invest more on digitization of service and delivery models. A last year's poll by Gallup - a global analytics firm-had $25 \%$ of Americans say that they or their family member had delayed treatment for a serious condition owing to the high costs of care ${ }^{1}$. Several European nations figure among the most expensive countries for hospitalization in the world-Monaco, Luxembourg, Norway, Switzerland, Denmark, Ireland, Netherlands, Sweden, Iceland, Finland and Austria ${ }^{2}$.

Realizing that adopting digital technologies is key to overcome these challenges and strengthen health systems, these countries started spending considerably on developing digital health innovations in the areas of telemedicine, mobile phone and applications, wearable devices, robotics, AI, genomics and big data.

In his acclaimed work The Creative Destruction of Medicine: How The Digital Revolution Will
${ }^{1}$ Bangalore, India. *sseethalakshmi2006@ gmail.com 
Create Better Healthcare, American cardiologist Eric J Topol has enumerated digital technologies as a driver for transformation of medical practice from the population-based approach to treating illness to individualized care. Mobile phones and computers converging with the Internet and rapid advancements in wireless remote sensors have enabled doctors in remote areas to monitor patients and provide timely intervention by gathering information in real time on their key health parameters.

Today, telemedicine is already proving to be a game changer in healthcare accessibility and affordability, with technologies like AI helping in accurate disease diagnosis, reducing a doctor's administrative burden, assessing a person's vulnerability to a certain disease and developing personalized medicines for individual patients by analyzing big data in medical research.

In the past 3 decades since the first surgical robot PUMA-that assisted a neurosurgical biopsy in 1985-ushered in an era of minimally invasive procedures, robotics has come to be integral to the present healthcare landscape. From the world's most widely used robotic surgical system da Vinci to radio-surgical robotic system CyberKnife's use in oncology to the SOCRATES tele-collaboration system, robots have become more precise and autonomous. Today, they are being used not just in a range of surgical procedures but for providing mobility to the disabled, aiding patients get out of beds and stand up, interacting with patients and reminding them of medications. As microbots are helping in diagnosis and treatment of chronic diseases, other kinds of robots are disinfecting hospitals and delivering medications and laboratory specimens inside premises.

The widespread use of robots in healthcare is proving to be essential in eliminating human error in procedures, improving patient care and reducing costs but their safe and secure deployment is something that cannot be ignored. Patient's trust is also a key challenge in largescale integration of robotics as not many trust a robot to perform complex procedures.

In the field of medicine, the first information technology intervention has been in the digitization of medical records. We now realize the critical role played by electronic health records and health information systems in eliminating medical errors, evidence-based decision making, automating and streamlining clinical workflow and improving treatment outcomes. The past several years have seen a steady growth in the adoption of electronic health records system across the world, of course with varying levels of integration.

The late 1970s and early 1980s saw a remarkable progress in genetics and ushered in the molecular biology revolution, with the new rapid DNA sequencing technique developed by Frederick Sanger in 1977 proving to be a turning point. Sanger's successful genome sequencing of a simple bacterial virus paved the way for sequencing of complex organisms and culminated with the beginning of the "Omics" revolution in the 1990s that saw the launch of the Human Genome Project-that mapped the entire human genome in 13 years. The sequencing of the complete human genome and its open access have since enabled identification of a host of genes associated with various diseases, efforts to correct faulty genes, establish susceptibility to diseases and choose most appropriate and effective drugs for treatment.

In the following decades, we have seen similar international initiatives such as the Human Cell Atlas, Human Brain Project and most recently the Global Virome Project.

The same time also saw something gaining extraordinary popularity across the world-the phenomenon of Quantified Self that implies self-tracking and using personal health data to enhance quality of life using wearable devices such as smart watches. The extremely convenient technology that has now become a part of everyday life of millions focuses on disease prevention and helps people live healthier, reduces the burden on health systems and the costs incurred. The global wearable device market has exploded since Fitbit launched the first such device in 2009, with global shipments touching 336.5 million units in $2019^{3}$.

What is relatively new but being looked as a powerful tool with a potential to immensely benefit healthcare is the Internet of Medical Things (IoMT), a network of interconnected medical devices and applications. It is proving to be of much help in both clinical and non-clinical fronts-monitoring a patient's vitals, assisting doctors with intuitive dashboards instead of them physically operating ICU devices and monitors, and relying less on nurses for recording of patient information. The technology is also being used in connecting ambulances with hospital information systems, tracking people movement in hospital premises and providing real-time information on queues to suggest appointment rescheduling. 
While all these developments have been stepping stones towards inching close to the dawn of the digital healthcare era we are witnessing today across the world, the COVID-19 pandemic has proved to be the single biggest trigger for accelerating digital transformation, all the more so in India. The viral outbreak has unveiled like never before alarming inadequacies of our healthcare infrastructure-shortage of beds, doctors and nurses, testing labs, heavy dependence on imports for medical equipment, testing kits and protective wear, low health insurance penetration, poor disease surveillance, lack of robust health data and rural inaccessibility to healthcare.

As India feels an urgent need for a major intervention in its healthcare, digital technology has already started gaining prominence as a game changer. Coupled with the government's push to technology driven healthcare in India, there has been an astounding response to COVID-19 in less than 5 months from the private enterprise like startups and hospitals, higher technical and scientific institutions in terms of developing new innovations in telemedicine, diagnostics, fitness and wellbeing apps, telehealth, video consultation, m-health and wearable sensors.

If the COVID crisis saw some of the country's major hospitals and health-tech startups to launch a nationwide telemedicine platform Swasth, it also witnessed startups joining hands to initiate project StepOne for people without smartphones and internet, enabling consultations with volunteer doctors through an automated interactive voice response system.

Most major hospitals in the country now offer virtual OPD services and many plan to make a majority of their OPD consultations virtual in the coming years. It is simply amazing to see how telemedicine is facilitating evaluation, diagnosis and treatment of patients using technologies such as phone and video call, chat platforms such as WhatsApp, Facebook and Skype, email and mobile apps. More doctors are now getting digitized images of patients, X-rays and blood reports through satellite-based communication links. Interacting with patients, consulting other doctors and suggesting appropriate treatment through video conferencing is becoming the norm. The convenient and user friendly system can also connect rural health centres to big hospitals in cities to facilitate quality patient care, providing a long-term solution for India's fragmented healthcare structure.
With just 4500 ICU specialists in the country, med-tech startups like Cloudphysician is leveraging technology to make ICU services available in remote locations. Just imagine a critical care team of intensivists and nurses in Bengaluru seamlessly connecting with patients in ICUs hundreds of kilometres away in Bihar through real-time audio, visual and electronic means. The platform has helped over 10,000 critical patients and manages over 300 ICU beds across 20 hospitals in eight states.

Secure mobile chat applications like Microsoft's Kaizala have enabled doctors do virtual rounds of ICU patients from wherever they are, even from an airplane, besides keeping ICU teams connected with shared lab reports and updates.

Likewise, digital technologies are taking diagnostics to the next level. Pathology is witnessing a digital leapfrog from conventional clinical mode with many diagnostics labs going completely virtual in terms of services, testing and reports.

Artificial Intelligence-driven diagnostic platforms have revolutionized cancer screening. It is amazing to learn how an AI-driven diagnostic platform-Niramai-is coming to the rescue of women with symptoms of breast cancer in the backward districts of North Karnataka. The platform uses patented thermal image processing and machine learning algorithms for accurate breast cancer screening. It is radiation free, nontouch and pain free unlike mammography.

We know how medical imaging is key to accurate diagnosis of a disease. Digital radiology is further pushing the levels of accuracy and has been accepted well by the medical community. It facilitates better assessment of disease as the size and contrast of the images produced on the computer screens can be digitally adjusted and shared with other departments and hospitals. The increasing integration of AI is medical imaging is helping in accurate radiology assessments and reduce errors besides enhancing efficiency with minimum input by aiding radiologists, with pre-screened images having identified features. AI-integrated digital radiology could be of immense use in oncology-from thoracic imaging, abdominal and pelvic imaging, detecting colonic polyps, identifying calcifications in breast, predicting brain tumours and assessing response to radiation therapy.

The months following the lockdown in March have seen a sharp surge in the number of home health service providers stepping in to fill 
the critical gap in healthcare delivery as millions of patients struggled to get appropriate medical help. At a time when hospital OPDs were shut and strict travel restrictions were in force, these companies offered a wide array of services ranging from doctor consultation, nurses and attendants to critical care, physiotherapy and medical equipment to lab tests, elderly, newborn and mother care to counselling. Amid huge shortage of hospital beds and doctors in India, home healthcare acts as a force multiplier and is set to change the present hospital-based healthcare.

The COVID lockdown also saw a boom in the sales of online medicine retailers with critical medicines being delivered to the doorsteps of millions of patients across the country.

Behind all this has also been the Government of India's proactive push to digital healthcare in the recent years. A host of technology-driven initiatives have been proposed and started by the government to make healthcare more effective, accessible and affordable-from digital framework like National Health Stack, National Digital Health Blueprint, National e-Health Authority, National Mission on Interdisciplinary Cyber Physical Systems, National AI Mission to Telemedicine Practice Guidelines issued in March this year. The eSanjeevani telemedicine platform recently recorded 2 lakh consultations across 23 states. Initiatives like e-Rakt Kosh that aims to integrate and streamline blood banks across all the states will play a major role in ensuring urgent blood availability to patients.

COVID-19 saw the Aarogya Setu app playing a key role in risk assessment and contact tracing besides being the central point in accessing all pandemic-related information and care. Connected to the app is web portal Aarogya Setu Mitr that allows users to access telemedicine consultations, testing services and home delivery of medicines.

The recently announced National Digital Health Mission envisages an integrated healthcare platform for India and has the potential to transform the sector. The mission envisages creating a personal health ID for all citizens, digitization of health records, registry of doctors, health facilities, e-pharmacy and telemedicine services. In all this it is important to ensure that accelerated digital adoption must not result in temporary substandard solutions or compromise with data privacy and protection. Taking a swift step in this direction, the government has already proposed a draft framework and standards to safeguard confidential health data collected under the mission.

While the country is taking steady steps towards a digital healthcare revolution, we must remember that digitization is facilitative and cannot substitute the much needed core structural reforms in public healthcare, most importantly in primary and preventive healthcare. India's public health spending is just over $1 \%$ of its GDP, one of the lowest in the world ${ }^{4}$. We have a total of 7,13,986 government hospital beds, which amounts to 0.55 beds per 1000 people as per the National Health Profile 2019 data, out of which just $5-8 \%$ are ICU beds. We have a single doctor for 1445 people in the country $^{5}$. We know that almost none of our district hospitals have EHR system and our primary health centres lack bare minimum physical infrastructure-enough beds, labour rooms, medical equipment, a computer with internet connection and even regular electricity and water supply. The government hospitals and dispensaries have very little ICT infrastructure, which is limited to a few large public hospitals in select metros.

There is little doubt that digital technologies hold the promise of bridging India's widening rural-urban divide but the current scenario makes it a far-fetched reality. India's 70\% population lives in rural areas having a low internet penetration of just $20 \%$ today, and only $7 \%$ of Indian households have access to broadband, almost all in cities ${ }^{6}$. There have been inordinate delays in the implementation of phase one and two of BharatNet, the flagship rural broadband project, despite repeated assurances by the government ${ }^{7}$.

For rural India to fully tap the potential of digital revolution, significant investment to boost digital infrastructure is a necessity. The access to digital technologies are also severely restricted in millions of rural homes still without electricity or facing long outages daily.

The pace with which digital health is evolving and the myriad ways it is helping transform healthcare indeed reaffirms that technology will be the fulcrum of a healthy future. Though with COVID-19 taking millions of lives across the world so far and predictions of more such zoonotic outbreaks in the coming years, the key question at this time is how can we leverage digital technologies to prepare ourselves for future pandemics?

We have seen how digital technologies can facilitate strategy and response to a global pandemic in the ways in which countries successful 
in maintaining low COVID-19 infection and mortality rates have adopted and integrated these technologies for pandemic planning, surveillance, testing, contact tracing, quarantine and healthcare. Countries like South Korea have demonstrated how digital technology can be integrated into government's containment and mitigation response.

The risk of zoonotic diseases is increasing with growing population, as we raze habitat for farmland and other development activities, travel more to explore remote forests, hunt, eat and trade wildlife, increasing the chances of a "spillover"-a virus jumping from an animal to a human. More than 260 viruses are known to infect humans, a tiny fraction of the unknown viruses that represent almost $99.9 \%$ of potential zoonoses which mostly remain undetected until they cause disease in humans ${ }^{8}$.

As witnessed during COVID-19, digital technology has an immense potential to support data-based epidemiological intelligence in accurate assessment of disease burden, tracking disease activity in real time, in identifying cases and clusters of infection, rapidly trace contacts, monitor travel patterns and enable large scale public messaging.

Technologies such as AI and big data can facilitate tracking of people, rapid diagnosis and risk prediction. New innovations like data dashboards can be extremely useful in collating public health data and visually display disease burden. Web and cloud-based cameras used in China and high-performance thermal cameras have facilitated rapid screening. Digital contact tracing apps increase tracing scale and speed to another level. GPS, cellular networks and WiFi provide location data that can be used to monitor people movement and identify potential hotspots. Online media, social media and chat platforms have played a key role in disseminating information and updates to the public about the disease $e^{9,10}$.

International initiatives such as The PREDICT Project and Global Virome Project have been intensively using digital technologies in detecting and identifying thousands of viruses to understand future threats.

As it becomes increasingly clear that the future of public health is 'digital', countries across the world must align their strategies to adopt and integrate disruptive digital innovations to make healthcare more seamless, effective and accessible. More so with deadly viral threats knocking at our doors more often, we cannot afford to wait any longer.

ENDS.

\section{Publisher's Note}

Springer Nature remains neutral with regard to jurisdictional claims in published maps and institutional affiliations.

Received: 31 August 2020 Accepted: 3 September 2020 Published online: 27 September 2020

\section{References}

1. Saad L (2019) More Americans delaying medical treatment due to cost. Gallup. https://news.gallup.com/ poll/269138/americans-delaying-medical-treatment-duecost.aspx

2. McDermott J (2018) Top 15 most expensive countries to be hospitalized in world. Finder.com's analysis of WHO's 2011 Health Costs Data for 189 countries. https://www. finder.com/most-expensive-countries-to-be-hospitaliz ed-in

3. Worldwide Quarterly Wearable Device Tracker report (2020) International data corporation. https:// www.idc.com/getdoc.jsp?containerId=prUS461221 20\#: :text=10\%20Mar\%202020-,Shipments\%20of\%20 Wearable $\% 20$ Devices $\% 20$ Rea ch $\% 20118.9 \% 20 \mathrm{Mil}$ lion\%20Units\%20in\%20the,for\%202019\%2C\%20Acc ording $\% 20$ to $\% 20$ IDC\&text=For $\% 20$ the $\% 20$ entire $\% 20$ year $\% 2 \mathrm{C} \% 20$ vendors, million $\% 20$ units $\% 20$ shipped $\% 20$ in $\% 202018$

4. National Health Accounts Estimates for India 2018. https ://main.mohfw.gov.in/sites/default/files/NHA_Estim ates_Report_2015-16_0.pdf

5. Doctor Count in India Below WHO Norm (2019) Telegraph. https://www.telegraphindia.com/india/docto r-count-in-india-below-who-norm/cid/1720533

6. Kumar A (2019) The promise and reality of digital technologies in bridging the rural-urban divide. Observer Research Foundation. https://www.orfonline.org/exper t-speak/promise-reality-digital-technologies-bridgingrural-urban-divide-56959/\#: :text=Global\%20Advisory $\% 20$ Board-, The $\% 20$ promise $\% 20$ and $\% 20$ reality $\% 20$ of\%20digital,bridging\%20the\%20rural\%2Durban\%20 divide\&text $=$ Digital\%20innovation $\% 20$ has $\% 20$ the $\% 20$ potential,pieces $\% 20$ of $\% 20$ the $\% 20$ economic $\% 20$ pie

7. Aryan A (2020) BharatNet phase two: about $7.45 \%$ of 1.5 lakh gram panchayats service ready so far. The Indian Express. https://indianexpress.com/article/technology/ tech-news-technology/bharatnet-phase-two-only-7-45per-cent-of-1-5-lakh-gram-panchayats-service-ready -6225281/\#: :text=Under\%20BharatNet\%20phase $\% 20$ two $\% 2 \mathrm{C} \% 20$ against, made $\% 20$ service $\% 20$ ready $\% 20$ so $\% 20$ far.\&text $=$ The $\% 20$ progress $\% 20$ in $\% 20$ states $\% 20$ such,two\%20is\%20among\%20the\%20worst

8. Carroll D et al (2018) Global Virome Project. Bulletin of the World Health Organization. https://www.who.int/ bulletin/online_first/BLT.17.205005.pdf 
9. Budd Jobie et al (2020) Digital technologies in the public health response to Covid-19. Nat Med 26(8):1-10. https ://doi.org/10.1038/s41591-020-1011-4
10. Whitelaw S, Mamas MA, Topol E, Van Spall HGC (2020) Applications of digital technology in COVID-19 pandemic planning and response. Lancet. https://doi. org/10.1016/S2589-7500(20)30142-4

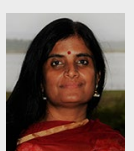

S. Seethalakshmi is a seven time award winning journalist and the former city editor of The Times Of India in Bengaluru. Having 17 years of journalism experience, she has covered and written extensively on politics and governance, science, health, education, environment, women and child issues and urban development. She is the recipient of International Visitor Leadership Program - the US Government's premier professional exchange programme. In her present capacity as the co-head of the think tank of HCG-South Asia's leading cancer hospital network, she is engaged in public policy initiatives in healthcare, governance and livelihood. She also continues to write for prominent national publications. decade in mainstream journalism during which he has held various editorial positions in some of the country's leading newspapers-The Times of India, The Indian Express and The Statesman. He was the deputy news editor of The Times of India, Delhi and Bengaluru, in his last assignment. Rahul was selected by the US Department of State for the prestigious Foreign Press Reporting Tour on "Emerging Women Entrepreneurs in America" as a prelude to the Global Entrepreneurship Summit held in India in 2017. He was also one of the two journalists invited from India to attend the Global Smart City Conference in Singapore in 2015. He continues to write on a range of issues for major news publications across India, writing extensively on COVID-19 most recently.

Rahul Nandan is a senior journalist and Chief Mission Officer of Antardhwani-an independent think tank of India's leading cancer care provider HCG-that works in the areas of healthcare, education, livelihood and governance. He has spent over a 